\title{
Illuminating the Voices of Sports Leaders in the COVID-19 Pandemic: A Conduit for Sports Development in the Sports Science School at UTech, Ja.
}

\author{
Van Der Rowe ${ }^{1}$ \\ Faculty of Science and Sport, University of Technology
}

https://orcid.org/0000-0001-5658-8139

\author{
Accepted: April 4, $2021 \quad$ Online Published: April 4, 2021
}

https//doi.org/10.30655/besad.2021.34

\begin{abstract}
The research used a phenomenological approach to examine the lived experiences of lecturers and students in the COVID-19 pandemic in the sports science school at the University of Technology, Jamaica. The results indicated that both lecturers and students endured physical and mental discomforts (e.g., weight gain and stress) in the transition to online learning. Through proactive leadership by the Head of School to put measures in place to alleviate some of the issues, sport science studies could continue with improved percentage passes throughout the period.
\end{abstract}

Keywords: Sports science studies, phenomenology, virtual learning

\footnotetext{
${ }^{1}$ Corresponding Author: Van Der Rowe vanderroweg@gmail.com

Faculty of Science and Sport, University of Technology, Jamaica, Kingston, Jamaica, W.I.
} 


\section{Introduction}

Sport is a driving force that has enabled persons to actively participate in different types of exercise and physical activity, which researchers have shown to offer positive benefits physically and psychologically (Clevinger, Petrie, Martin, \& Greenleaf, 2020). Clevinger et al. (2020) further explained that humans' health, whether physical or mental, seems to be a concern that many countries are taking measures to correct. It has been well established that sports and physical activity are the determining factors that offer significant benefits to the human body physically, psychologically, and emotionally. Can the physical, psychological, and emotional well-being that sports offer be achieved in indoor isolation? Well, a study titled "Benefits of outdoor sports for society. A systematic literature review and reflections on evidence", conducted by Eigenschenk, Thomann, McClure, Davies, Gregory, Dettweiler, and Inglés (2019), found that the advantages of outdoor sports also go beyond its involvement in a non-natural surrounding; have been illustrated by experts from various fields in this context.

Sport is a term that is sometimes used for physical activity and vice versa. It is focused on the inclusion of all types of physical activity that promote or enrich a person's health and well-being physically or mentally from the participation in organized or casual activities. This helps to promote social relationships or to achieve results through different competitions at varied levels (Eigenschenk et al., 2019). Eigenschenk et al. (2019) further explained that outdoor sporting activities could magnify social interactions in developing young people's interpersonal skills, violence reduction, and an environment of active citizenship. Furthermore, outdoor sporting activities provide a natural and socially interactive environment, which provides many benefits to indoor sporting activities.

Hsiao-Pu, Stone, Churchill, Brymer, and Davids (2017) published research titled "Physical and Emotional Benefits of Different Exercise Environments Designed for Treadmill Running," in which they examined the effect of participating in indoor sport and physical activity. The research results indicated that while participating in the activities, participants could get physical benefits, but the impact that green physical activity would have was missing. Hsiao-Pu et al. (2017) further explained that despite significant portions of the world living in cities where access to green physical activity and sport is limited. The evidence remains that green physical activity and sports provide more excellent all-round benefits in terms of socialization, psychological and emotional development through the interaction with nature. Do virtual sports affect sport science students, and how does this impact the relationship between school culture and student performance? Well, according to Baena-Arroyo, Garcia-Fernández, Gálvez-Ruiz, and Grimaldi-Puyana (2020), they believe that there are positive and negative benefits for sport science students engaging in virtual sport and physical activity classes. However, it does affect the overall engagement, quality, performance, and attitudes towards participation.

The atmosphere influences students and demonstrates the critical effect of school culture or climate on students' outcomes. Usually, school climate refers to the establishment of social and interactive engagement of the school atmosphere, with students, families, and teachers all contributing to the running of a school and the treatment of the physical environment, making it a community phenomenon greater than any one person's experience (Cleveland \& Sink, 2017). In other words, the student's individual experiences are aggregated into a school-wide climate, both influencing the academic and behavioral performance of students. School climate can be defined as the composition of students' social interactions, behavior, shared values, cultural attitudes, personal beliefs, a system of values, and the cultivated norms. This school climate helps shape and guide the delivery of the teaching and learning process, the achievements, and the institution's day-to-day running (Cleveland \& Sink, 2017).

Throughout the last eight months, athletes and sports-related areas such as sports studies have started to move operations to a virtual modality that engages practical activities from home. This sort of forced initiative has become the expected means of communication for both coaches, students, and athletes to get support, instructions, and the execution of plans. The COVID-19 pandemic has severely impacted every fiber of sports. The adaptation to virtual and blended engagement has become very difficult and poses many risks to individuals in the sporting industry. Despite this, many sporting organizations have established safety guidelines for athletes, coaches, and other sport-related areas, such as the students 
doing sport studies and persons wanting to exercise. The sporting industry and other sports-related areas such as sports schools and fitness trainers must consider the unprecedented situation that faces us. Ministries must try to support elite athletes and other areas surrounding sports to ensure that the industry does not totally fall apart, but at the same time, practitioners must put the health of athletes and students first.

Just as sporting bodies and elite athletes are trying to get accustomed to the new normal, this is the same for the sports science students at the Caribbean School of Sports Science at UTech, Ja. The students are now being engaged in distance learning and blending modalities which may come with their challenges. This paper seeks to Illuminate the Voices of Sports Leaders in the COVID-19 Pandemic: A Conduit for Sports Development in the Sports Science School at UTech, Ja.

\section{Methods}

\section{Research Approach and Design}

This study utilized an interpretive phenomenological approach that seeks to generate an understanding, explore and interpret the lived experiences of selected lecturers and students at the Caribbean School of Sport Sciences (CSOSS) at the University of Technology, Jamaica (UTech. Ja.). Since not all researchers have the same belief system, researchers have to consider their philosophical assumptions about the nature of acquiring facts and knowledge. The prism through which one views the world plays a significant role in performing and perceiving research data while assessing beliefs, behaviors, decisions, and the research process (Patton, 2017). Ontology concerns the nature of being; it is the study of reality. At the same time, epistemology aims to show us how to obtain truth awareness (Patton, 2017). Also, scholars belong to various philosophies; thus, different paradigms of analysis are based on ontological and epistemological views (Patton, 2017).

Qualitative analysis requires thick, descriptive text rather than numbers (Patton, 2017). Questions in qualitative research are broad and open-ended so that participants' stories are properly derived, and sense-making can occur without the researchers' prior assumptions (Patton, 2017). In this form of study, personal interactions are present so that meaning is addressed. As stated earlier, the purpose of this interpretive phenomenological study is to describe and interpret the lived experiences of selected lecturers and students in the CSOSS at the UTech. Ja.

\section{Interpretative Phenomenological Analysis}

This Interpretative Phenomenological Analysis (IPA) was used to analyze selected lecturers and students' experiences. The research incorporated an IPA as a methodological approach. Due to frustrations over the overemphasis on the significant impact that can be gained in psychological research using a qualitative approach, IPA was implemented as a particular research technique (Smith, 1996 as cited in Goodall, 2014). Smith (1996), as cited in Goodall (2014), argued that there was a need for change to improve research in the area and thereby deepen the psychological understanding. Goodall (2014) emphasizes that IPA's emphasis is on analyzing deep-rooted personal experiences of people and understanding and interpreting the entire phenomena to shape their reality sensibly.

The meaning behind this phenomenon is to understand them, people are deeply interested in reality and are consistently reflecting and reliving their personal experiences. There are two critical goals for researchers using IPA as an approach (Alase, 2017). First, participants are allowed to share their experiences and then try to get an in-depth understanding of the phenomenon through an insiders' view. Secondly, an analysis of the situation is conducted to understand the individuals' concerns regarding the phenomena (Alase, 2017). Within philosophy, the IPA foundations are told by three main areas: phenomenology, hermeneutics, and idiography (Goodall, 2014; Alase, 2017). To get a better understanding of the emphasis and objectives of IPA, it is necessary to keep this in mind. The meaning 
behind them can be used to increase understanding of the experiences of selected lecturers and students.

\section{Sample}

Noon (2018) points out that researchers should aim to recruit a reasonably homogeneous sample when introducing IPA; these persons are not necessarily different, substantially regarding their demographic details. The CSOSS is a relatively small department with five full-time lectures, two program directors and one head of school, and several part-time lecturers. The research focus on four full-time lecturers, the sports science program director, and the head of school to get these lecturers' lived experiences in the COVID-19 pandemic. The research also sought to understand student experiences throughout this process by selecting students from the three undergraduate sport science Programmes that CSOSS offers. Five students participated in the study: two students doing sports management (SM), two students doing athletic training (AT), and one student doing coaching.

The researcher conducted structured interviews with four lecturers, the program coordinator, the head of school, two students doing SM and AT, and one student doing coaching as the data source. The research interview sought to understand participants' views, experiences, and beliefs that empowered them during the COVID-19 pandemic and its impact on the sport science studies at UTech, Ja. Interviews provided the researcher with data that have a deeper meaning and understanding of the issues through a qualitative process rather than the quantitative method. The lecturers, in this case, provided data regarding the actual implementation of the changes and how they differ from the standard delivery of module content. They highlighted the challenges that they faced in this mode of delivery and its possible impact on students and performance. The program director provided data on the restructuring of some modules, re-socialize students, resolving issues and the pandemic's impact, and this mode of delivery on the students and lecturers.

The head of school provided data about the changes from a school-wide perspective and a university perspective. The head of school also provided data on staff performance during the pandemic versus other periods. At the same time, the students are needed to provide data on their perspective of the pandemic changes. Students also provided data on how the pandemic impacted them and how the changes to their modules have affected their learning style, performance, and grades.

\section{Data Analysis}

This analysis was a very time-consuming process that started with the first interview until the research was completed. After each interview, the researcher completed memoing or reflective writing to record the interviewer's thoughts, comprehension, and reactions. Maxwell (2013) and Kirillova (2018) outlined several stages to be followed in conducting qualitative data analysis; these are the stages that were used to analyze the data. The stages included reading and re-reading, exploratory commenting, which examined the themes that emerge while trying to find linkages between themes. The researcher proceeded to the following interview while searching for similar patterns across cases. The lack of memoing was associated with Maxwell and Noon (2013, ;2018) as a type of Alzheimer's disease researcher. They warned researchers that it would not be possible to recall important details of the interview without mentioning those elements.

\section{Ethical Considerations}

Ensuring that a respectful and trustworthy approach underpinned the research process is very important throughout the design phase and, of course, the implementation stage. The researcher ensured that proper ethical standards were maintained concerning approval (from the university and participants), confidentiality, and the reduction of any harm to the participants. Both lecturers and students had the requisite competence to agree to participate and thoroughly and truthfully updated by using a Participant Information Sheet regarding all aspects of the study. Before deciding to participate, the participants were given sufficient time to discuss and process the request to participate. Before the start of each interview, the participants were required to provide their consent in writing. The 
participants were also required to confirm that they are comfortable with the information presented in the permission letter, acknowledge that they would participate voluntarily, and ensure they understood that responses would be anonymized.

\section{Results}

The results from this research are a collection of the voices from students, lecturers, the program director, and the head of school. The results have shared a deep perspective and understanding of their experiences studying, lecturing, and administrating sports studies at the university. The results are presented based on the four themes that emerged from the data analysis: The effect of the pandemic, the school's adaptation to the pandemic, the positive impacts of virtual learning, and the challenges faced throughout the pandemic.

\section{The Effect of the Pandemic}

The mental and physical impact of the pandemic seems to be general issues that have been affecting people throughout the COVID-19 pandemic. This research showed that majority of the participants, both administrators, lecturers, and students suffered from weight gain, increased stress levels, and the isolation caused decreased social interactions. Participant S1 explained that "I felt that I was at a brink state of depression. I was not sure if I was going to make it. I felt down, to be honest". One of the contributing variables to the state of depression and stress that many staff and students felt was being isolated from social life and personal interactions. Participant L1 and L2 respectively explained that on the "mental side now, there was some amount of adjusting to do because of a change of going out being up and about and I am that kind of person generally.... "mental state, there is a certain level of stress... adjusting to the teaching environment and teaching modality".

The physical impact on many staff and students, as stated above, was weight gain due to the inability to exercise and eating more being at home. Participant A2 stated that "I found that at home, doing all this mental work, I was eating more than I normally would." While participant S1 added similar sentiments "in terms of eating, you eat a lot when you are home." There were a few participants that believed the pandemic had not affected them mentally nor physically. Participant S2 believes that the pandemic had no physical impact "honestly you will find this strange or whoever reading this or any student, but it has not impacted me physically'. While participant L4 explained that "mentally, I have grown a bit stronger, because of family situations and I have to be the strong one."

\section{The school's adaptation to the Pandemic}

One of the points expressed strongly by the lecturers interviewed and the administrators was the school's readiness before the onset of the pandemic that led to virtual learning in March 2020. The participants agreed that the school had established a plan for a hybrid approach and an alternative to that in case the virus had spiraled out of control and we had to go fully online. Praises for this type of response were placed squarely at the feet of the Head of School. The participants, including the students, thought the school's readiness was good, and the leadership was excellent. Participant L2 explained that "I think she did what she could given the circumstances... and continue to try to provide leadership... she provided open means of communication, seeking feedback from lecturers as to what is happening, how things are happening". While L1 explained that "I would think she did a very good job, she was very communicative, supportive, decisions were made and whatever decisions were made, even higher than she was she communicated those promptly," L1 further explained that:

interactions on WhatsApp, in whatever was needed to be communicated to us rapidly, even when, for example, sanitization things were not provided. She provided them herself for us to use. So, if I were to rate her possibly out of ten (10), I would be close to nine (9). 
Most of the lecturers praised the Head of School's efforts that she did her best based on the situation. As explained by participant L3, "the leader has been welcoming, and she has been helpful, she ensures that whatever needs to be done, is done. She also let us know what was happening. So, we were not in a vacuum". The lecturers sharing their experience during the pandemic indicated that they had to write module planners and get their modules on Moodle in anticipation of going entirely online. Despite the school's readiness, some lecturers believed that the general university's readiness affected the smooth transition to virtual learning because the university did not have the necessary software and platforms for virtual learning. Participant L4 indicated that "what I realized though the fact that how we had responded, I think one of the beliefs that had occurred was delayed from all of it at the top of the university." Participant L1 also explained that "from the standpoint of online, I possibly think, while university tried to provide a platform that everybody could have used and be comfortable with. There were many glitches with it; nonetheless, students were unable to get on to the platform".

\section{The Positive Impacts of Virtual Learning}

Despite some of the issues associated with virtual learning, the participants agreed that there were positives that came out of this experience. Participants believed that the pandemic helped them improve their critical thinking through the mode of delivery and the types of assessments given. Participant A1 explained that:

If you hear anybody complain that the exam was too hard. It is because it is a lot of cognitive questions, and it is not something that you can just recall and circle. So that is good; it is stretching us in terms of how we set our questions, stretching the students. Even in terms of presentation, we ask them to do more presentations now, and you realize how interesting it is when they go and seek out the information.

The pandemic allowed some students and lecturers to work and study in a more relaxed environment at home. Participant A1 explained that "the title of the assessments is more geared to smoother or less stressful for students. So, I think they are doing better. I think they are doing better, their grades look much better". Also, more time was available for lecturers and students to prepare lectures and do assignments. Both lecturers and students got the opportunity to explore more specialized software and platforms to make teaching and learning more creative. Participant L4 indicated that "my methodological approach has to be different to reach the students and try to enthuse them, bring them to a higher level." This also required students and staff to learn about these platforms and tools, in turn helping to improve their technological skills.

Participant L2 indicated that "it has been an adjustment, from the work point of view, adjusting to teaching online or teaching hybrid." Lecturers and students indicated the reduction of traveling time and cost (rent, bus fare, gas, and printing). Some lecturers even believed that the pandemic had helped them to improve their overall performance as a lecturer. On the other hand, a few students believed they saw improved grades as a result of many improved variables. Participant A1 explained that "I think they are doing better, their grades look much better," participant A1 further explained that:

I am going to put it down to the fact that the students are getting case studies to analyze. They are not so stressed because of the assessment, the time to do their assessment, and for the ones who complain, they are getting more cognitive questions, so they have to think more which is what we want them to do.

\section{The Challenges}

According to participant L1, "the school numbers would have gone down; I think that persons being unsure, not having access to the internet devices." All the participants could relate to internet connectivity issues, and some lecturers had students with device issues but based on the lecturers' responses, it was not widespread. Participant L1 further explained that students "not being sure of either having a device whether you will be able to have the connectivity throughout the entire period based on where you are situated." Participant A2 indicated that with "regards to access to the internet, I didn't 
receive any wholesale report of students not having access. I know for one module we had a student who didn't have access to an electronic device to complete her assessment".

One of the most expressed issues in many of the interviews is that live online practical classes were challenging, and some students could not master skills using this medium. According to participant S3, "when it comes to online classes, the practical was kind of difficult. It is easier to conduct classes face to face. That was the real issue for me". did internet connectivity issues also reduce the video sessions' quality when students and lecturers turned their cameras on. Students expressed their concerns about not mastering critical practical skills and missing out on the opportunity to get invaluable practical experience. Participant S1 explains her anxiety about the lack of practical exposure:

honestly, it made me wonder, if we do not have these activities coming up, then what purpose are we serving as sport science students? If we do not have sports in science, how does it make any sense? And learning these things online or not doing the hands-on is not the same thing. You have to practice what you are learning. And if you are only getting a little bit of the practice, and a whole heap of the theory is not exciting. And you have students falling asleep in your class or just not wanting to show up at all. So we as sport science students feel it, and we do not know how to express our feelings.

Lecturers and students are hopeful that these practical face-to-face experiences that were missed will not affect students' long-term. Participant L4 indicated that

the practical component of it I realized is slow, and a slight decrease in the way in which the practical aspect of the skills was executed would have been the cause of the lack of face to face practice from a theoretical standpoint.

Another issue with the live online practical sessions was getting and using the correct equipment that is required. Participant S4 explained that:

You could have the right, and proper equipment like for track and field we had to do throws and I did not have a shot put or discuss, I had to improvise and make one (1) and you do not have the proper weight. Those things could alter your techniques, as in how you approach the proper one to the makeup you have at home.

Students and lecturers use the opportunity to be very creative in making their sporting implements for online classes. The anxiety remains whether using improper equipment will impact how students learned the skills and execute them in real-life situations. Participant S4 indicated that "I had a small space to work with. If I had UTech, I would have the big fields, so I had to adjust my technique to fit in that environment that I was in". Participant S4 further explained that:

sport has a lot of practical aspects; it is mainly practical aspects. So if you cannot get it there, it might affect you in the future, or you have the theory part, the practical part might let you down in the future

Despite the equipment challenges with online practical sessions, facilities and space was a big problem for lecturers and students whose living accommodations did not fully allow them to execute the skills. Participant L1 explained that "at intervals when you asked persons to turn their camera on, you could have seen that their environment would not have been conducive to the teaching-learning process for several different reasons." The initial onset of the pandemic and virtual learning was not managed well by most students who felt stressed and were unable to cope. The lecturers indicated that they motivated students while having many inspirational talks with students to help them cope.

\section{Documentary Review}

This process sought to examine semester 12019 grades when normal operations were taking place at the university versus semester 12020 when Jamaica and the university were fully affected by the pandemic. During this period, several changes were made to sports science modules and their delivery 
to ensure the continuation of the teaching and learning process. The researcher sought and obtained the faculty's validation reports with percentage passes from the modules offered in semesters 1 of 2019 and 2020. However, both semesters 1 and 2 had a few outstanding modules up to validation where grades are validated. Based on the analysis and comparison of the performance passes for the modules offered during semester 12019 and 2020 in the Caribbean School of Sports Sciences, the results indicated a 3.6\% increase in the overall performance of the school module pass rate in semester 1, 2020.

\section{Discussion and Conclusion}

This chapter aims to clarify, refine and examine the results of this study concerning the existing body of literature. However, very little research has been done looking at the effects of the pandemic on sport studies. The purpose of this study was to ascertain the impact of the COVID-19 pandemic on sports science studies at the University of Technology, Jamaica, through the lived experiences of the administrators, lecturers, and students. The participants were able to share their detailed experiences of the pandemic to include positives, negatives, and areas they can now improve on going forward. The discussion results in this chapter are presented using the research questions.

\section{To what extent did sport science studies at UTech, Ja. change and adapted to the COVID 19 pandemic?}

The results of the research suggest that sport science studies at UTech, Ja. changed completely to facilitate the COVID-19 pandemic and ensured that the Programmes could continue and student learning was not affected. A study published by Domokos et al. (2020) titled "Being a student at the Faculty of Sports and Physical Education in COVID- 19 Pandemic times - A moment in life" has strongly supported these research findings. Domokos et al. (2020) research suggested that their faculty shifted online entirely at the onset of the pandemic, which also saw a change in how sports modules were delivered in that institution. These were similar findings portrayed by this research. As a result of the change to fully online in September, CSOSS adopted a more frequent communication policy for administrators, lecturers, and students using WhatsApp, emails, and phone calls. Through this improved communication, information reached lecturers and students a lot quicker. The school also established a sanitization and equipment/facility protocol that changed how sport science students interact and participate in practical face-to-face sessions when they had to go on campus.

The school was very proactive in its approach to putting in two options before the full effect of the pandemic. Option A was to have a blended or hybrid approach where students would have virtual lectures and conduct practical sessions face-to-face; if the pandemic worsened, option B would have been put in place. Option B entailed a fully online delivery of both lecture and practical sessions. Domokos et al. (2020) strongly supported this approach, which indicated that they did not have a plan B, but they proceeded fully online. Based on lecturers' feedback, the hybrid approach was started but based on increases in COVID-19 cases and travel restrictions from the government; the university went fully online. Based on these research results, several initiatives were employed to facilitate the continuation of sports education during the pandemic.

\section{Module delivery and creativity}

When the onset of the pandemic brought on increased cases, the university went fully online; the lecturers interviewed in this research indicated that they used Moodle as a storage house for course materials and interactive assessments such as quizzes and course tests. While using Zoom and Blackboard collaboration to host synchronous classes with students. To ensure a high level of integrity for quizzes and tests on Moodle, some lecturers indicated that they employed security features and monitored students' screens during their quizzes and tests to ensure cheating did not occur. Going fully online meant that the process would be new to many CSOSS lecturers and students, as indicated in the results. The lecturers indicated that it was challenging to keep students engaged with the many distractions around them at home. Students that were interviewed also endorsed the fact that distractions were very real for them during online learning. Lecturers indicated that they had to be very 
creative to keep students constantly engaged by doing forum discussions, giving jokes, having students keep their cameras on, and even playing games with students.

Domokos et al. (2020) research supports the findings in this research that identified live virtual practical classes as the preferred method for lecturer and student engagement during this period. However, the results of this suggest that using differentiated platforms engaged students' interest and participation more. For the practical classes, both students and lecturers interviewed indicated that some level of creativity was needed. Practical classes were done online using three formats (1) live online practical classes where lecturers and students would turn on their cameras over Zoom or Blackboard collaboration and instructions given for how the practical class will be executed, students also indicated that they had to use family members as participants or clients in their live practical sessions to aid in the demonstration of skills or to practice the learned skills; (2) Pre-recorded practical classes, some lecturers were able to pre-record some of their practical classes in preparation for going entirely online while other lecturers were able to source YouTube videos to be watched live in the classes by students and lecturer which is followed by discussions and demonstrations; (3) class discussions followed by pre-recorded videos by students. Students were required to record themselves practicing/demonstrating the skills discussed in the class and at times teaching what they learned to other persons such as immediate family members that they were isolated with. Students and lecturers would then watch these pre-recorded videos in the classes and further corrections and discussions took place for each pre-recorded video. The results of this study suggest more creative methods were employed to engage students fully. In contrast, Domokos et al. (2020) study indicated that students were unwilling to turn on their mics and their cameras to participate in the classes.

For creativity in practical classes, students were required to create implements needed to learn and teach the practical areas. Students and lecturers indicated that the equipment was not readily available to all students because of the country's lockdown at one point, so students were guided in creating their implements. Another part of the creativity employed by CSOSS lecturers to ensure that sports studies could continue was the modification of personal living quarters by students to facilitate live practical classes. Based on the diameter of students living accommodations, the lecturer and the students were able to use the areas to execute the required activities. Students were also required to use whatever they had in their surroundings to aid in creativity and the delivery of the practical areas. This study also suggests that students were more active and willing to engage in the practical class. However, Domokos et al. (2020) also found that space was a problem for those students who chose not to participate, while lecturers in this research indicated that students were allowed to utilize their surroundings and the limited space they had.

\section{Assessments}

The results of this research indicated that many of the lecturers restructured their module assessment breakdown (MAB) to facilitate an online delivery. Several changes were made to assessment to include: (1) Case studies, (2) video analysis, (3) applied assessment (4) research papers. As stated earlier, the participants indicated no final exams were done; instead, students were given a final assessment in any of the above. Also, quizzes and tests were done mainly on Moodle with added security and the monitoring of student's screens.

As it relates to practical assessment, three formats were highlighted by lecturers and students through the interview process: (1) Live practical assessment, students would be given a particular topic to demonstrate/teach live over a video conferencing platform and would be assessed by the lecturer. The student, based on the topic, may execute it with family members' aid as participants or possible clients. (2) Face-to-face assessment, students were required to go on campus in small numbers for their practical assessments. (3) Pre-recorded practical assessment, students were given a particular task and asked to record the execution of the task and send the video recording to the lecturer for grading. 


\section{To what extent did the pandemic impact student performance?}

Assessing the pandemic's impact is a bit difficult in a phenomenological study; as a result, the researcher employed a documentary analysis of semester 12019 and semester 12020 to analyze changes in student performance. Despite the documentary analysis results, the participants all gave different perceptions of the impact of the pandemic on student's performance.

The administrators thought students did better in the pandemic in specific modules than they would have done usually. The administrator's perception is that the MAB had changed with no final exam and more research and application areas, which allowed students to do better. They also believed that studying in the comfort of their homes, less stress with studying for final exams, more creative forms of assessment, better distribution of grades, and less traveling afforded students more time for school work to aid in their performance.

The lecturer's perception is that students did well in the modules' theoretical aspects, but for modules that carried a practical component or that was fully practical, students performed below expectations. Lecturers believed that this performance could be attributed to many variables such as poor internet connection to allow for camera interactions, access to the required equipment, the necessary space in students' homes to execute practical sessions. Also, the ability to have personal face-to-face interactions for the demonstration and correction of skills. Lecturers indicated that students did a lot better in previous years at the practical aspect of various modules. According to participant L1 who tested the theory, the participant indicated that after a period of virtual practical classes because of the restriction in the early part of the semester, they ventured on campus after the restrictions had eased. He was surprised as to the level that the students were performing and what they knew. The participant indicated that students would have performed a lot better if they were engaged in face-to-face practical sessions, this was also supported by the other lecturers.

The students believed that their grades were not affected despite the challenges of transitioning from face-to-face classes to online. The student participants who were interviewed believed that despite the rigors of online learning, they had excellent lecturers in CSOSS that ensured that they could maintain their grades. All the students interviewed found virtual practical sessions difficult. However, they still believed that the areas where they did poorly resulted from their lack of interest in participating or the many distractions and time management issues that they faced, which was not a result of the pandemic. What students attributed to the COVID-19 pandemic is the isolation and the transition to online learning. They believed that if they were face-to-face with lecturers, they would have done a lot better because the lecturers could provide the type of correction, feedback, and assistance needed to grasp the skills fully. The research that was done by Domokos et al. (2020) also supported these findings that students prefer the regular face-to-face teaching and learning process.

The documentary review discovered that module passes had increased by $3.6 \%$ compared to its corresponding period in 2019. The general enrollment for the different modules offered during this period remained relatively the same. However, the documentary review only analyzed the percentage passes for an entire module and not individual student grades. As a result, this process could not give a true reflection of whether students' individual performances improved. Many variables could have impacted the module performances, such as the change in the type of assessment and the percentage weighting of different areas. Despite the variables that may have impacted module performances, it is clear that students' performances were impacted positively throughout the pandemic. The administrators, lecturers, and the documentary review all indicated that students' performance had improved compared to the 2019 corresponding period.

\section{What impact did leadership have on the program and performance of students in the pandemic?}

One of the high points of the results is the impact of leadership on students and the sport science studies at UTech, Ja. Students and lecturers praised the leadership efforts for their proactive move to put plans in place before the university announced the general initiatives. The Head of School (HOS) was able to get lecturers writing module planners to get all the CSOSS modules for that year on the university's learning platform, Moodle. The HOS also encouraged lecturers to utilize the services of 
recording sessions offered by the faculty. There was a significant improvement in frequent communications with staff and students to deliver university administration decisions. The results indicate that the leader had frequent meetings, involved staff in the decision-making process, sourced sanitizing aids for lecturers, met with students, structured the offerings of the programmes that could be done safely, and assisted in developing sanitization protocols for the equipment and facilities.

\section{Conclusion}

The general feelings from both staff and students are that the COVID-19 pandemic required a transition period that brought both physical and mental implications. The research results indicated that despite the challenges that staff and students faced during the pandemics' initial stages, they were able to proactively find creative solutions for the continuation of sport science studies. This continuation affects how sport science studies were offered (hybrid and fully online) through creative synchronous class engagement, critical thinking, applied theoretical assessments, creative technologically abled practical classes, and assessments. This new form of learning engagement for students created a perception from administrators who believed students improved their performance throughout the period, lecturers who believed that theoretical performance improved. At the same time, the practical areas could have been much better. The students believed that their grades remained the same compared to previous semesters, but they all agreed that online practical classes were difficult. However, they could have done a lot better if they had more engagement in face-to-face practical sessions.

In conclusion, the administrators and lecturers all believed that student's performance was impacted positively in different ways while increasing compared to previous semesters. The documentary review results also confirmed that module performance passes increased by $3.6 \%$ compared to its corresponding period in 2019. The documentary analysis was unable to determine what contributed to the increase because it only analyzed validation reports and not individual students results. The responses from administrators, lecturers, and students indicated that the increased performances could have result from changes in module assessment, module percentage weighting, and the mode of delivery. The HOS response to the pandemic was seen as very proactive in ensuring that CSOSS was ahead of the game in the preparation of the hybrid or fully online approach. The HOS leadership was seen as communicative and involved with regard to lecturers and students receiving timely information and guidance on the way forward.

\section{References}

Alase, A. (2017). The interpretative phenomenological analysis (IPA): A guide to a good qualitative research approach. International Journal of Education and Literacy Studies,5, 9-19. http://10.7575/aiac.ijels.v.5n.2p.9

Baena-Arroyo, J. M., García-Fernández, J., Gálvez-Ruiz, P., \& Grimaldi-Puyana, M. (2020). Analyzing consumer loyalty through service experience and service convenience: Differences between instructor fitness classes and virtual fitness classes. Sustainability, 12(3), 828. http://dx.doi.org/10.3390/su12030828

Bas, D., Martin, M., Pollack, C., \& Venne, R. (2020). The impact of COVID-19 on sport, physical activity, and well-being and its effects on social development. United Nations Department of Economic and Social Affairs. Retrieved on January 8, 2021, from https://www.un.org/development/desa/dspd/wp-content/uploads/sites/22/2020/05/PB_73. pdf

Cleveland, R. E., \& Sink, C. A. (2017). Student happiness, school climate, and school improvement plans. Professional School Counseling, 21(1) http://dx.doi.org/10.1177/2156759X18761898 
Clevinger, K., Petrie, T., Martin, S., \& Greenleaf, C. (2020). The relationship of sport involvement and gender to physical fitness, self-efficacy, and self-concept in middle school students. Physical Educator, 77(1), 154-172. http://dx.doi.org/10.18666/TPE-2020-V77-11-9228

Dabic, T., \& Stojanov, Z. (2015). Reflections on some validity and ethical issues in mixed methods research on investigating English language usage at IT departments in Serbia. Researchgate, 2-6. http://22.10.1016/j.asmart.2020.07.006

Domokos, C., Domokos, M., Mirică, S. N., Negrea, C., Bota, E., \& Nagel, A. (2020). Being a student at the faculty of sports and physical education in COVID- 19 pandemic times - A moment in life. Timisoara Physical Education and Rehabilitation Journal, 13(24), 45-50. http://dx.doi.org/10.2478/tperj-2020-0007

Eigenschenk, B., Thomann, A., McClure, M., Davies, L., Gregory, M., Dettweiler, U., \& Inglés, E. (2019). Benefits of outdoor sports for society. A systematic literature review and reflections on evidence. International Journal of Environmental Research and Public Health, 16(6). http://dx.doi.org/10.3390/ijerph16060937

Escamilla-Fajardo, P., Núñez-Pomar, M. J., Calabuig-Moreno. F., \& Gómez-Tafalla, M. A. (2020). Effects of the COVID-19 pandemic on sports entrepreneurship. Sustainability, $12,84-93$. http://dx.doi.org/10.3390/su12208493

Evans, A., Blackwell, J., Dolan, P., Fahlén, J., Hoekman, R., Lenneis, V., McNarry, G., Smith, M., \& Wilcock, L. (2020). Sport in the face of the COVID-19 pandemic: Towards an agenda for research in the sociology of sport. European Journal for Sport and Society

Goodall, D. (2014). An interpretative phenomenological study exploring designated teachers' experiences of supporting looked after children. Unpublished Doctoral dissertation, The University of Sheffield.

Harris, A. (2020). COVID-19 - School leadership in crisis? Journal of Professional Capital and Community, 5(3/4), 321-326. http://dx.doi.org/10.1108/JPCC-06-2020-0045

Hsiao-Pu Yeh, Stone, J. A., Churchill, S. M., Brymer, E., \& Davids, K. (2017). Physical and emotional benefits of different exercise environments designed for treadmill running. International Journal of Environmental Research and Public Health, 14(7), 752. http://dx.doi.org/10.3390/ijerph14070752

José Sá, M., \& Serp, S. (2020). The COVID-19 pandemic as an opportunity to foster the sustainable development of teaching in higher education. Sustainability.

Kirillova, K. (2018). Phenomenology for hospitality: Theoretical premises and practical applications. International Journal of Contemporary Hospitality Management, 30(11), 3326-3345. http://dx.doi.org/10.1108/IJCHM-11-2017-0712

Maxwell, J. A. (2013). Qualitative research design. Thousand Oaks, CA: SAGE Publications.

Mayende, G., Prinz, A., Maurice, N. G., Muyinda, B. P., \& Nampijja, D. (2016). Philosophical and ethical concerns in researching online learning groups. Conference on e-Learning, e-Management, and e-Services, 1-7.

Melo, L. A. M. (2016). Contributions of the hermeneutics of Paul Ricoeur to the phenomenological

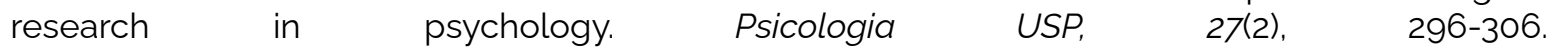
http://dx.doi.org/10.1590/0103-656420140071

Netolicky, M. D. (2020). School leadership during a pandemic: navigating tensions. Journal of Professional Capital and Community,5(3/4), 391-395. http://dx.doi.org/10.1108/JPCC-05-2020-0017

Noon, E. (2018). Interpretive Phenomenological Analysis: An Appropriate Methodology for Educational Research? Journal of Perspectives in Applied Academic Practice,6, http://dx.doi.org/10.14297/jpaap.v6i1.304 
Patton, C. M. (2017). Workplace conflict, management, and leadership implications: An interpretive phenomenology of medical imaging technologists. Unpublished Doctoral dissertation, Eastern University.

Peck, B., \& Mummery, J. (2019). Recovering the "individual" for qualitative research: an idiographic approach. Forum: Qualitative Social Research, 20(3), http://dx.doi.org/10.17169/fqs-20.3.3231

Wong, A. L., Ling, S., Louie, L., Law, So., R., Lee, D., Yau, F., \& Yung, P. (2020). Impact of the COVID-19 pandemic on sports and exercise. Asia-Pacific Journal of Sports Medicine, Arthroscopy, Rehabilitation, and Technology.

World Health Organization (2020). Mask use in the context of COVID-19. Retrieved January 8, 2021, from https://www.who.int/emergencies/diseases/novel-coronavirus-2019?gclid=CjoKCQiAyJOBBhD CARIsAJG2h5fXeGfH7IYtVy6MGb35ADWo4ULcyLey7uKbnOd4wLLL7_juAfBpsmkaAvepEALw_w $\mathrm{CB}$ 